\title{
A silent myocardial infarction in a diabetic patient after partial foot amputation: Case report and review of the literature
}

\author{
Adelaida Avino ${ }^{1}$, Cristian Radu Jecan ${ }^{1,2}$, Cristina-Nicoleta Cozma ${ }^{1,2}$, \\ Daniela Elena Gheoca-Mutu', Andra-Elena Balcangiu-Stroescu ${ }^{3,4}$, \\ Maria-Daniela Tanasescu ${ }^{5,6}$, Dorin Ionescu, ${ }^{5,6}$, Laura Raducu ${ }^{1,2}$ \\ ${ }^{1}$ Department of Plastic and Reconstructive Surgery, \\ "Prof. Dr. Agrippa Ionescu" Clinical Emergency Hospital, Bucharest, Romania \\ ${ }^{2}$ Discipline of Plastic and Reconstructive Surgery, Faculty of Medicine, \\ "Carol Davila" University of Medicine and Pharmacy, Bucharest, Romania \\ ${ }^{3}$ Department of Dialysis, Emergency University Hospital Bucharest, Romania \\ ${ }^{4}$ Discipline of Physiology, Faculty of Dental Medicine, \\ "Carol Davila" University of Medicine and Pharmacy, Bucharest, Romania \\ ${ }^{5}$ Department of Nephrology, Emergency University Hospital Bucharest, Romania \\ ${ }^{6}$ Department of Medical Semiology, Discipline of Internal Medicine I and Nephrology, Faculty of \\ Medicine, "Carol Davila" University of Medicine and Pharmacy, Bucharest, Romania
}

\begin{abstract}
The diabetic foot syndrome is the most common cause of diabetic patients hospitalization, being a challenging complication of diabetes. This is a case report of a diabetic patient who presented a silent myocardial infarction after partial foot amputation.

The patient presented normal troponin at admission, but after 21 days of hospitalization he acused a very slight pain in the chest and breathing difficulty. The troponin was $682 \mathrm{ng} / \mathrm{l}$. He was transfered immediately in the intensive care unit for specific treatment. The evolution of the surgical intervention was favourable even under the poor general status. The patient fully recovered after 10 days and left the cardiology department in a good clinical condition.

A silent myocardial infarction seems to occur with increased frequency in patients with diabetes, due to the cardiac autonomic dysfunction. It is highly important a quick diagnosis and a good management of the patient.
\end{abstract}

Keywords: myocardial infarction, diabetic foot, cardiovascular disease, troponin, amputation

\section{INTRODUCTION}

The diabetic foot syndrome or disease (DFD) is considered to be a feared disability, being the most common cause of hospitalization for diabetic patients. The syndrome can be described by the triad of neurophaty, ischemia and infection. Diabetic foot ulceration affects almost $15 \%$ of all patients during their lifetime (1). It appears in all types of diabetes, more frequently in males and after the age of 60 (2). Just $20 \%$ of the patients with foot ulcera- tions go on to need an amputation. But more than $85 \%$ of the amputations come after diabetic foot ulcers (1) and worldwide every 30 seconds one lower limb is lost because of this syndrome (3). The incidence of DFD is growing due to its predisposing factors. The main pathology involved is the diabetic peripheral neuropathy, followed by the peripheral arterial disease. Other risk factors that should be highlighted are: the limited ability to ove the joint, the foot deformities, earlier ulcerations or amputations, but also reduced visual acuity, chron- 
ic renal disease, duration of diabetes longer than 10 years and uncontrolled hyperglycemia (4).

The mortality rate after diabetic foot amputation is increased to up to $80 \%$ after 5 years, which is bigger than that seen in most malignancies. Furthermore, DFD is responsible for long period of hospitalization compared with any other chronic complications of diabetes mellitus (3).

Up to $60 \%$ of all ulcerations in diabetic patients are the cause of neuropathy, while $45 \%$ of the foot ulcers appeared due to mixed neuropathic and ischemic factors. The most common form of neuropathy in diabetic patients is the distal bilateral symmetrical neuropathy, which usually appears in the lower limbs, meanwhile the upper limbs can be affected in some cases too (4). The patients are unable to detect any traumatic lesion that appears in the affected site, due to the loss of sensation, so many woulds go unobserved.

Regarding atherosclerosis in diabetic patients, it is similar to that seen in nondiabetics, but it developes early and has an accelerated progression. The macrovascular disease in patients with diabetes can be characterized by coronary artery, cerebrovascular and peripheral vascular disease (1). The coronary artery disease appear two to three decades earlier in diabetic patients compare with nondiabetics. It is considered to be one of the main causes of death in diabetic patients among with the stroke. Recent studies shows that $20-50 \%$ of patients with diabetes mellitus have silent coronary artery disease. It can be observed after a silent myocardial ischemia. More that $75 \%$ of ischemic episodes are asymptomatic, based on continuous electrocardiographic recordings (5).

Silent myocardial infarction, defined as evidence of myocardial infarction (MI) on the electrocardiogram in the absence of history of MI, appears in almost $50 \%$ of the patients diagnosed with MI. It is considered that both clinical and silent MI are associated with poor prognosis. Silent MI was first described in 1949. In 1959, it was presented in the Framingham Heart Study. It is associated with an increased risk of heart failure independent of HF risk factors. In the general population, $0,3 \%$ to $4,8 \%$ of the patients have silent MI. But, the elderly, diabetic patients and women are known to have a higher prevalence of up to $15 \%$. Silent MI is associated with increased risk of reinfarction, other coronary heart disease and sudden cardiac death (6).

\section{EXPERIMENTAL}

A 61-year-old obese male patient presented to the Department of Plastic Surgery of „Prof. Dr.
Agrippa Ionescu" Emergency Clinical Hospital with wet gangrene of the right foot. The patient had diabetes mellitus type II insulin-dependent, with sustained uncontrolled hyperglycemia and peripheral neuropathy. The 4th and the 5th toes were amputated in another hospital 6 months before. He was a non-smoker, with arterial hypertension, coronary artery disease and retinopathy. Prior admission wound cultures and foot radiography were performed.

At that time lab results revealed serum haemoglobin $(\mathrm{Hb}) 9.5 \mathrm{~g} / \mathrm{dl}$, serum creatinine $1.13 \mathrm{mg} / \mathrm{dl}$, urea $56 \mathrm{mg} / \mathrm{dl}$, Na $136 \mathrm{mmol} / 1$, serum albumin 3.16 $\mathrm{g} / \mathrm{dl}$, erythrocyte sedimentation rate (ERS) 115 $\mathrm{mm} / \mathrm{h}$ and fibrinogen $551 \mathrm{mg} / \mathrm{dl}$. His vital signs were within normal limits, blood pressure was $140 / 70 \mathrm{mmHg}$, heart rate -75 beats/min, temperature $-36,5{ }^{\circ} \mathrm{C}, \mathrm{O}_{2}$ saturation $-99 \%$. Partial foot amputation was performed.

On day 21 of admission the patient acused a very slight pain in the chest and breathing difficulty. The troponin I (TnI) was $682 \mathrm{ng} / \mathrm{l}$ and the NTproB-type natriuretic peptide (NT-proBNP) 7131 $\mathrm{pg} / \mathrm{ml}$. He was transfered immediately in the intensive care unit for specific treatment. After two days in the intensive care unit he was transfered in the cardiology department for further investigations. The evolution of the surgical intervention was favourable even under the poor general status. The patient fully recovered after 10 days.

\section{RESULTS}

On the foot radiography, osteoarthritis of the first toe and osteomyelitis of the second and third toe could be seen (Fig. 1). Antibioterapy was started with Ceftriaxone $-2 \mathrm{~g} \mathrm{x} 2 /$ day and Vancomycin $1 \mathrm{~g} \times 2$ /day for 10 days according to the positive results of wound swab cultures, our patient having Proteus mirabilis and Enterococcus faecalis.

Partial foot amputation was decided. An antiseptic dressing was applied which was changed every day. After 7 days, secondary suture was performed.

As a postoperative complication, in the context of the clinical signs and symptoms, the electrocardiogram (Fig. 2) suggested the presence of a first degree atrioventricular block (PR interval $-219 \mathrm{~ms}$ ), minor left bundle branch block and an antero-septal myocardial infarction being confirmed by the following parameters: sinus rhythm, heart rate -83 $\mathrm{bpm}$, cardiac axis - 0 degrees, poor $\mathrm{R}$ wave progression in V1, V2 and V3 leads, slight ST-segment and $\mathrm{T}$-wave abnormalities in V5 lead, increased 
cardiac enzymes- TnI $682 \mathrm{ng} / 1$ (1.5-50 ng/1 nor$\mathrm{mal})$, NT-proBNP $7131 \mathrm{pg} / \mathrm{ml}(0-300 \mathrm{pg} / \mathrm{ml}$ nor$\mathrm{mal})$, D-dimer $1428.88 \mathrm{ng} / \mathrm{ml}(0-500 \mathrm{ng} / \mathrm{ml}$ normal).

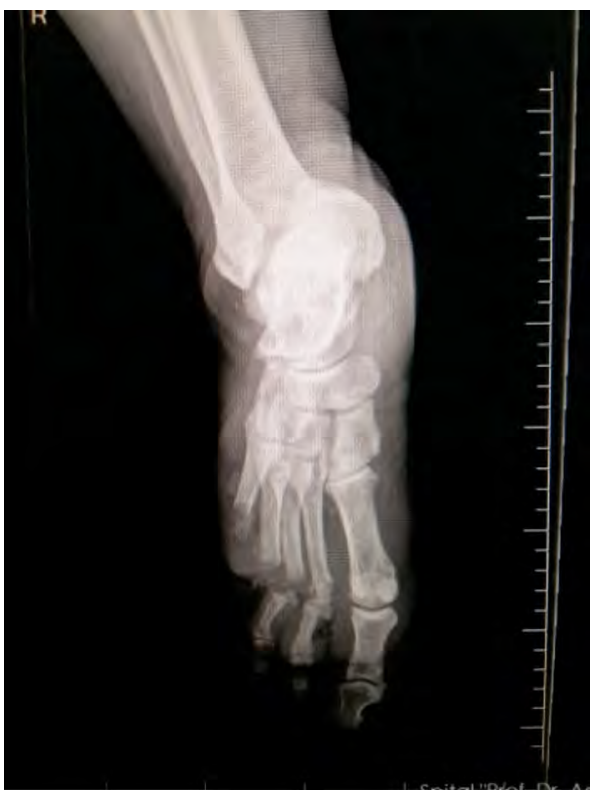

FIGURE 1. The foot radiography with osteomyelitis and osteoarthritis

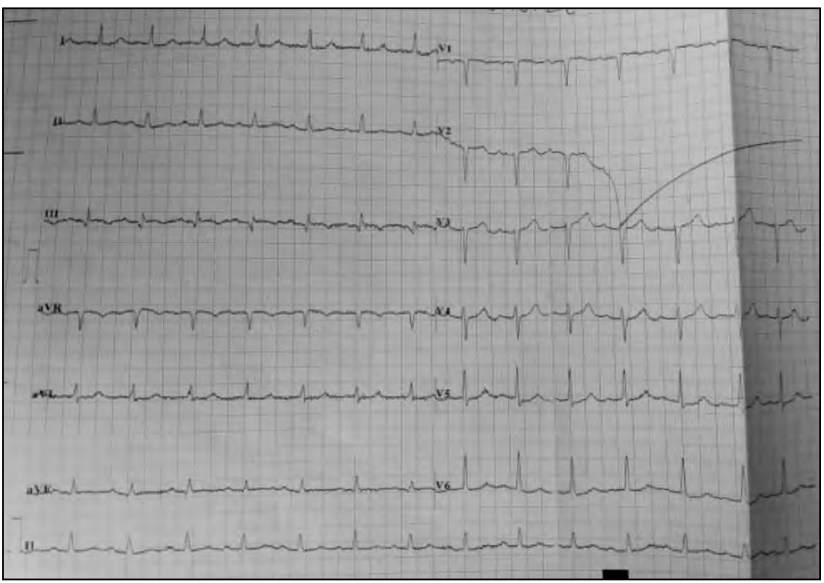

FIGURE 2. The electrocardiographic changes on day 21 of admission

Hemodynamic stabilization was done in the intensive care unit and just after about 48 hours he was admitted to the cardiology depatment for further treatment.

In the cardiology department, coronary angiography was performed and revealed $70-80 \%$ stenosis of proximal left main, $80 \%$ stenosis of anterior descending artery in segment II, 70\% stenosis of circumflex artery in segment I. Moreover, the echocardiography described a nondilated left ventricle, concentric, ejection fraction $=45 \%$, apical $1 / 3$ anterior wall akinesia, mild mitral regurgitation, severe aortic stenosis, grade II tricuspid regurgitation, free pericardium. Because there was no lesion identi- fied to be responsible for the acute coronary syndrome, no interventional revascularization was performed. The evolution was favorable, without angina and with remission of the heart failure symptoms. The patient has indication for surgery aortic prosthesis and aorto-coronary bypass which he refuse.

\section{DISCUSSIONS}

Diabetes mellitus is a growing worldwide health concern, with an estimated global prevalence of 366 million people by the year 2030 (3). Diabetic foot ulcer is considered to be the most costly and harmful complication in diabetic patients (7). Diabetic foot syndrome can be described by two types of ulcers such as neuropathic ulcers and neuroischaemic ulcers. In general, neuropathic ulcers appear on the plantar aspect of the foot under the metatarsal (8). Our patient had distal bilateral symmetrical neuropathy. His feet were warm and well perfused, but with dry skin.

On the foot radiography, outbreaks of osteoarthritis (OA) were observed. OA anddiabetes mellitus often co-exist in older adults. It is observed that there is a higher prevalence of arthritis in diabetic patients , almost $52 \%$ compared to those without it, $27 \%$. In general, OA in elderly diabetic patients is a source of greater disability and economic burden. Lately, OA has been associated with systemic metabolic disorders frequently seen in diabetes mellitus, showing that diabetes in and of itself determine it, independently of obesity or aging. Age and obesity contribute to development of cartilage and bone abnormalities by different pathways (9). Moreover, hypertension and dyslipidemia, two of the main risk factors for diabetes have been suggested to contribute to the development of OA, narrowing the blood vessels and producing subchondral ischemia. All these lead to cartilage degradation (10). Hyperglycemia may also directly affect the cartilage health in OA by means of advanced glycation end-products and peripheral neuropathy (9).

In diabetic foot syndrome, the sensory nerve fibers are affected in almost all the cases. Motor nerve fibers are occasionally affected too and muscle denervation can occur. In the early stages of the disease, the muscle power is conserved, but a slightly muscle weakness in the toe extensors can be observed. As the disease advances, muscle weakness progresses, affecting small muscles in both feet modifying the normal foot dynamics and pressure distribution, but also joint stability leading to foot deformities (4). Pressure distribution distur- 
bance in gait determines the preulcerative lesion in the neuropathic foot, the callus. The callus presses on the soft tissues below and cause foot ulceration (8). The neuropathic foot is also described by the loss of the deep sensation, such as vibration perception and proprioception leading to sensory ataxia. According to Wagner-Meggitt classification (4) our patient had a grade 4 wound more exactly gangrene in the forefoot. He told that the lesion progressed in one year from superficial ulcer to a deep one. He was treated in another hospital for six months.

Infections in diabetic patients are usually dangerous, potentially life threatening. An ulcerated, infected wound in diabetic foot is limb threatening and must be taken cared rigorously. Debridement, oral antibiotics and regular dressings are indicated for superficial, infected lesions $(11,12)$, commonly caused by gram-positive bacteria. When the bones, tendons or muscles are affected, it is considered to be a deep infection, that in almost all the cases is polymicrobial caused by gram-positive bacteria, gram-negative bacteria and anaerobes (13). Initially empirical antibiotherapy can be administrated, but once the results of the would culture are released, specific antibiotics must be prescribed (1). Due to the fact that our patient presented with wet gangrene of the left foot, with one gram-negative bacteria and another gram-positive bacteria, surgical debridement was decided, removing all the devitalized tissues, sloughed tendons and affected bones. So a partial foot amputation was performed. Postoperative, the antiseptic dressing was changed every day in aseptic conditions for 7 days, prior the secondary suture.

Mortality rate after lower limb amputation are extremely high (14) and it is significantly related to age and pre-amputation morbidity (15). Older age, proximal amputation levels and multi-morbidity, renal disease and peripheral vascular disease are all associated with a higher rate of mortality after amputation (16). In literature, 1 year after amputation leads to a mortality rate of $22 \%$ in a population that included partial foot amputation and $52 \%$ in pa- tients with transtibial and more proximal levels (14).

On day 19 after the surgery, our patient was diagnosed with myocardial infarction, but clinically he acused just a very slight pain in the chest and breathing difficulty. The electrocardiogram and the increased cardiac enzymes confirmed the diagnosis.

Coronary artery disease is the main cause of mortality in diabetic patients. The MI is often asymptomatic. It is detected in an advanced stage when it becomes clinically manifest. In diabetes, the morbidity and mortality are significantly high, once the coronary artery disease is symptomatic (17). In recent studies, the diagnosis of silent MI was based on the presence of Q wave on electrocardiogram, in most of the cases. In the rest of the cases, R-wave reduction or abnormalities of the ST segment and/or $\mathrm{T}$ wave were considered the proof of the MI (18). The prevalence of silent MI is up to $10 \%$ in diabetic patients with peripheral neuropathy (19) and almost $37 \%$ in patients with peripheral artery disease with silent MI detection by dipyridamole thallium scintigraphy (20). The proportion of silent MI is particularly high in patients with cardiac autonomic neuropathy $(18,21)$. In addition, age, prior cardiovascular disease, micro/macroalbuminuria (22) and diabetes duration are notably predictive of silent MI (23).

\section{CONCLUSIONS}

We highlight this case due to the fact that incidence of silent myocardial infarction is increasing in patients with diabetes, due to the cardiac autonomic dysfunction. It is highly important a quick diagnosis and a good management of the patient. These are achievable and can be life saving. The best approach is prevention, especially in diabetic patients with foot amputation.

\section{Acknowledgement}

All authors have same contribution for this article.

5. Prasad DS, Kabir Z, Devi KR. Subclinical atherosclerosis and silent myocardial ischaemia in patients with type 2 diabetes: a protocol of a clinicoobservational study. Open Heart 2014;1:e000100. doi:10.1136/ openhrt-2014- 000100

6. Qureshi et al. Silent MI and Heart Failure. JACC VOL. 71, NO. 1, 2018 Jan 2018, 71 (1) 1-8

7. Yazdanpanah L, Nasiri M, Adarvishi S. Literature review on the management of diabetic foot ulcer. World J Diabetes. 2015; 6(1):37-53.

\section{BIBLIOGRAFIE}

1. Pendsey SP. Understanding diabetic foot, Int J Diabetes Dev Ctries, 2010;30(2):75-9.

Rathur HM, Boulton AJ. The diabetic foot. Clin Dermatol. 2007; 25:109-120.

3. Madanchi et al. Who are diabetic foot patients? A descriptive study on 873 patients. Journal of Diabetes \& Metabolic Disorders 2013, 12:36

4. Amin N, Doupis J. Diabetic foot disease: From the evaluation of the "foot at risk" to the novel diabetic ulcer treatment modalities. World $\mathrm{J}$ Diabetes. 2016;7(7):153-64. 
8. Edmonds ME, Foster AV. Diabetic foot ulcers. BMJ. 2006; 332(7538):407-10.

9. Piva SR et al. Links between Osteoarthritis and Diabetes:Implications for Management from a Physical Activity Perspective. Clin Geriatr Med. 2015 February; 31(1): 67-87.

10. Zhuo Q, Yang W, Chen J, Wang Y. Metabolic syndrome meets osteoarthritis. Nat Rev Rheumatol. 2012 Dec; 8(12):729-37.

11. Răducu L, Balcangiu Stroescu A.E., Stanescu I.I., Tanasescu M.D., Cozma C.N., Jecan C.R.,Badita D.G. Use of polyhexanidine in treating chronic wounds. Septembrie 2017, Revista de Chimie - Bucharest- Original Edition- 68(9):2112-2113

12. Răducu L., Cozma CN, Balcangiu Stroescu A.E., Avino A., Tanasescu M.D., Balan D.G.,Jecan C.R.. Our experience in Chronic Wound Care with Polyurethane Foam, March 2018, Revistade Chimie -BucharestOriginal Edition, 69(3)(539-758).

13. Lipsky BA. Medical treatment of diabetic foot infections. Clin Infect Dis. 2004 Aug 1;39 Suppl 2:S104-14.

14. Fortington LV et al. Short and Long Term Mortality Rates after a Lower Limb Amputation. Eur J Vasc Endovasc Surg. 2013 Jul;46(1):124-31.

15. Kristensen MT, Holm G, Kirketerp-Møller K, Krasheninnikoff M, Gebuhr P. Very low survival rates after non-traumatic lower limb amputation in a consecutive series: what to do? Interact Cardiovasc Thorac Surg. 2012 May;14(5):543-7.

16. Thorud JC, Plemmons B, Buckley CJ, Shibuya N, Jupiter DC. Mortality After Nontraumatic Major Amputation Among Patients With Diabetes and Peripheral Vascular Disease: A Systematic Review. J Foot Ankle Surg. 2016 May-Jun;55(3):591-9.
17. Wackers FJ et al: Detection of silent myocardial ischemia in asymptomatic diabetic subjects: the DIAD study.Diabetes Care. 2004 Aug; 27(8):1954-61.

18. Valensi $P$, Lorgis $L$, Cottin $Y$. Prevalence, incidence, predictive factors and prognosis of silent myocardial infarction: a review of the literature. Arch Cardiovasc Dis. 2011 Mar;104(3):178-88.

19. Niakan E, Harati Y, Rolak LA, Comstock JP, Rokey R. Silent myocardial infarction and diabetic cardiovascular autonomic neuropathy. Arch Intern Med, 146 (1986), pp. 2229-2230

20. Nesto RW, Watson FS, Kowalchuk GJ et al.Silent myocardial ischemia and infarction in diabetics with peripheral vascular disease: assessment by dipyridamole thallium-201 scintigraphy. Am Heart $\mathrm{J}$, 120 (1990), pp. 1073-1077

21. Balcangiu-Stroescu AE, Tănăsescu MD, Diaconescu A, Răducu L, Bălan DG, Mihai A, Tănase M, Stănescu II, lonescu D. Diabetic nephropathy: a concise assessmen of the causes, risk factors and implications in diabetic patients, Revista de Chimie (Bucharest), 69, No.11, 2018, pg: 3118-3121

22. Balcangiu-Stroescu $A E$, Tănăsescu MD, Diaconescu $A$, Răducu L, Bălan DG, Mihai A, Tănase M, Stănescu II, Ionescu D. Nutritional intervention in patients with diabetic renal disease - a brief presentation. Rev. Chim. (Bucharest), 69, No.11 , 2018, p: 3178-3182

23. Burgess $D C$, Hunt $D$, Li $L$ et al.Incidence and predictors of silent myocardial infarction in type 2 diabetes and the effect of fenofibrate: an analysis from the Fenofibrate Intervention and Event Lowering in Diabetes (FIELD) study. Eur Heart J, 31 (2010), pp. 92-99 